\title{
The Role of Vitamin D in Cognitive Disorders in Older Adults
}

\author{
Jake Gold, Abdullah Shoaib, Gopinath Gorthy and George T Grossberg \\ Department of Psychiatry and Behavioral Neuroscience, St Louis University School of Medicine, St Louis, MO, US
}

DOI: https://doi.org/10.17925/USN.2018.14.1.41

$\mathrm{T}$ he physiological effects of vitamin D on calcium/phosphorus metabolism have been well studied since its discovery in the early 20th century. With recent advances in cellular and molecular biology, its role in maintaining normal brain functions and the protection of neurons via maintenance of cellular homeostasis, immune regulation, modulation of synaptic structure and function are more clearly known. Recently, its deficiency is increasingly implicated in major neurocognitive disorders including Alzheimer's disease, Parkinson's disease, and vascular dementia. Older adults are particularly vulnerable not only because vitamin D deficiency becomes more prevalent with aging, but they also are often complicated with other comorbid illnesses. This article reviews the role of vitamin D in maintaining normal brain functions, and implications for vitamin D deficiency in cognitive disorders.

\section{Keywords}

Vitamin D, 25-hydroxyvitamin D, geriatric, cognition, dementia, neurocognitive disorder, Alzheimer's disease, Parkinson's disease, vascular dementia

Disclosure: Jake Gold, Abdullah Shoaib, Gopinath Gorthy and George T Grossberg have nothing to declare in relation to this article.

Review Process: Double-blind peer review.

Compliance with Ethics: This article involves a review of the literature and did not involve any studies with human or animal subjects performed by any of the authors.

Authorship: All named authors meet the criteria of the International Committee of Medical Journal Editors for authorship for this manuscript, take responsibility for the integrity of the work as a whole and have given final approval for the version to be published.

open Access: This article is published under the Creative Commons Attribution Noncommercial License, which permits any non-commercial use, distribution, adaptation and reproduction provided the original authors and source are given appropriate credit. (C) The Authors 2018 .

Received: January 15, 2018

Accepted: March 29, 2018

Citation: US Neurology. 2018;14(1):41-46

Corresponding Author: George T Grossberg,

Department of Psychiatry and Behavioral Neuroscience, Saint Louis University, 1438 South Grand Boulevard, St Louis, MO 63104, US. E: george.grossberg@health.slu.edu

Support: No funding was received in the publication of this article.
Recent research suggests that vitamin D plays an important role in preventing and/or decreasing the risk of a variety of neurodegenerative disorders in addition to its well-established role in bone and calcium metabolism. It is estimated that $40-80 \%$ of geriatric patients have low levels of serum vitamin D. ${ }^{1}$ In this article we review the latest research that examines the role of vitamin $D$ in neurocognitive disorders in the geriatric population. We will look at the epidemiology of vitamin D deficiency with particular reference to older adults, the common sources of vitamin $D$, the reasons for vulnerability of older adults toward hypovitaminosis $\mathrm{D}$, and the role of vitamin $\mathrm{D}$ in brain metabolism. Finally, we will conclude by summarizing new clinical data on the role of vitamin $\mathrm{D}$ in major neurocognitive disorders (MND) in older adults.

\section{Epidemiology}

Although there is some debate over what the reference range serum levels for vitamin $D$ (25-hydroxyvitamin D) are, two of the more common definitions of the lower limit of normal are a serum level less than $75 \mathrm{nmol} / \mathrm{L}\left(30 \mathrm{ng} / \mathrm{mL}\right.$ ) or less than $50 \mathrm{nmol} / \mathrm{L}(20 \mathrm{ng} / \mathrm{mL}) .{ }^{2}$ Regardless, low serum concentrations of vitamin $\mathrm{D}$ are very common in the general population. It is approximated that in the United States over $40 \%$ of adults have serum vitamin D levels less than $20 \mathrm{ng} / \mathrm{mL} .{ }^{2}$ This is even higher in certain subpopulations. For instance, approximately $60 \%$ of African Americans have vitamin D deficiency, and worldwide it is estimated that $40-80 \%$ of geriatric patients have low levels of serum vitamin D. ${ }^{1}$

In patients with cognitive difficulties, hypovitaminosis $\mathrm{D}$ is quite prevalent, with estimates ranging from $70-90 \% .{ }^{3}$ Similarly, low vitamin D is prevalent in other pathologies such as depression, major neurocognitive disorder, and psychosis. One study noted that nearly $72 \%$ of psychogeriatric patients had low vitamin D. ${ }^{1}$ There is some preliminary data to suggest that early-stage idiopathic Parkinson's disease (PD) is also associated with low vitamin D levels, with one study citing that nearly $70 \%$ of patients with PD studied had low vitamin D. ${ }^{2}$

\section{Sources of vitamin D}

There are a few foods that are natural sources of vitamin D such as salmon, cod liver oil, and mackerel..$^{1,2}$ In addition to naturally occurring sources of vitamin D, many commercial food products are fortified with vitamin D, particularly dairy products such as milk, cheese, and yogurt. ${ }^{1}$ There are also a plethora of over-the-counter vitamin supplements containing vitamin D. ${ }^{1}$ However, the major source of vitamin D for most people is endogenous production necessitating sunlight. ${ }^{1}$ When sunlight, particularly ultraviolet B radiation, interacts with the skin, it facilitates the conversion of 7-dehydrocholesterol (7-DHC) in the skin to 25-hydroxyvitamin D. ${ }^{2}$ Therefore, any factors that reduce direct sunlight exposure, such as use of high-sun protection factor (SPF) sunscreen, having more melanin in the skin, staying indoors, or geographical location, could cause decreased levels of vitamin D. ${ }^{2}$ 


\section{Risk factors for hypovitaminosis D in older adults}

As people get older, skin thickness and 7-DHC content decrease, leading to decreased capacity of the skin to produce vitamin D. The skin loses more than $50 \%$ of its ability to produce vitamin D at 70 years of age compared with 20 years of age. ${ }^{1}$

Multiple pathologies can contribute to the functional decline that occurs in the gastrointestinal system with aging. Atrophic gastritis, altered gastric absorption, and decreased hepatic metabolism are all common among older adults. Fat malabsorption from celiac disease, Crohn's disease Whipple's disease or gastric bypass cause impaired absorption of vitamin D, a fat-soluble molecule. Similarly, because the liver is a site of vitamin D metabolism, hepatic dysfunction can also cause low vitamin D levels. ${ }^{2}$

In addition, chronic kidney disease can alter calcium metabolism and can ultimately cause decreased serum concentration of

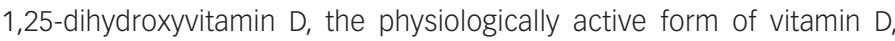
in older adults. ${ }^{3}$ Medications, such as glucocorticoids and antiepileptic drugs also impair vitamin D synthesis. ${ }^{2}$

\section{Clinical implications}

Individuals that have hypovitaminosis D as a result of a genetic defect in vitamin $\mathrm{D}$ binding protein have been shown to have an increased risk of all-cause mortality. ${ }^{4}$ Low vitamin D levels have been associated with a plethora of different conditions, such as obesity, osteoporosis, muscle weakness, cardiovascular disease, and a variety of MNDs. ${ }^{2}$ Specifically, vitamin $D$ has been associated with incidence of cognitive decline and mild cognitive impairment (MCl), Alzheimer's Disease (AD), PD, and vascular dementia (VaD). ${ }^{5-12}$

Multiple studies have demonstrated a correlation between lower serum vitamin D levels and memory and cognitive decline in older adults. 10,11,13 In a study that compared patients with $\mathrm{MCl}$ and low vitamin D levels with a group of similar patients with normal vitamin D levels, the group with lower vitamin D had more memory complaints, as measured by the Memory Complaint Questionnaire (MAC-Q). Other studies have also demonstrated this relationship between hypovitaminosis $\mathrm{D}$ and $\mathrm{MCl}{ }^{6}$

Although hypovitaminosis $D$ is not an established risk factor for $A D$ or $\mathrm{PD}$, multiple studies have found a correlation between either disorder and low serum vitamin D concentrations. ${ }^{5,7,8}$ Other studies have failed to replicate these findings. ${ }^{14,15}$ However, there is evidence to suggest that this relationship is causal based on the finding that mutations in the vitamin D receptor $(V D R)$ gene have been shown to correlate with development of $A D$ and $P D .^{16-21}$

Beyond the correlation with disease incidence, low vitamin D status may be a prognostic indicator of cognitive and mood outcomes in PD. One study found that lower vitamin D levels were related to a small but significant increase in severity of motor dysfunction in patients with PD after 36 months. ${ }^{8}$ Higher vitamin D levels in patients with PD have also been associated with improved cognition and mood. ${ }^{22}$ The finding that certain VDR gene polymorphisms are associated with poor cognitive function in patients with PD provides further support for this hypothesis. ${ }^{18,23}$

Vascular dementia also has a connection with vitamin $D$ status Multiple epidemiological studies on VaD have shown association with hypovitaminosis D. ${ }^{9,24,25}$ One recent retrospective study utilizing neuroimaging failed to find any correlation. ${ }^{12}$ The significance of a correlation is controversial as there may be confounding variables related to lifestyle that may be responsible for this relationship. In order to investigate a causal relationship, one study examined whether vitamin D status was independently related to hypertension, a major etiology of VaD. The study found that low vitamin D was associated with higher blood pressure, but they could not support a causal relationship as ischemic stroke incidence had a significant relationship with observed hypovitaminosis D, but not with presumed hypovitaminosis $\mathrm{D}$ due to genetic predisposition. ${ }^{26}$

\section{Role of vitamin $\mathrm{D}$ in the brain}

Vitamin D plays a central role in normal brain functions and the protection of neurons via maintenance of cellular homeostasis, immune regulation, and modulation of synaptic structure and function. Vitamin D acts mostly via the VDR, which is a nuclear receptor that combines with the retinoid $X$ receptor $(\mathrm{RXR})$ to regulate gene transcription. ${ }^{27}$ Because vitamin $\mathrm{D}$ has a role in normal brain functions, which will be outlined below, we propose that hypovitaminosis D may contribute to cognitive decline with old age, $A D$, VaD, and PD. (See Figure 1).

\section{Cellular homeostasis}

In older adults there is an increase in influx of ionized calcium $\left(\mathrm{Ca}^{2+}\right)$ into neuronal cell bodies, which interferes with the action potentials necessary for long-term potentiation (LTP) and the formation of new memories. ${ }^{28}$ The increase in $\mathrm{Ca}^{2+}$ activates both ryanodine receptors (RYRS) and calcineurin, causing additional release of endogenous calcium and longterm depolarization (LTD) (i.e., the erasing of memories), respectively. The increase in $\mathrm{Ca}^{2+}$ has been attributed to increased expression of the L-type voltage-dependent Ca ${ }^{2+}$ channel (LVSCC); vitamin D3 downregulates the expression of multiple subunits of LVSCCS, and therefore vitamin D status mitigates the major cause of $\mathrm{Ca}^{2+}$ dysregulation with aging. ${ }^{28,31}$

The effects of vitamin $\mathrm{D}$ on $\mathrm{Ca}^{2+}$ regulation are essential to the prevention of cognitive decline and $A D$. One hypothesis even attributes the onset of $A D$ to an initial vitamin $D$ deficiency that begins a positive feedback loop between $\mathrm{Ca}^{2+}$ dysregulation and amyloid- $\beta$ (A $\beta$ ) plaque formation. This idea is supported by the discovery that $\mathrm{Ca}^{2+}$ dysregulation results in increases in the formation of $A \beta$ plaques; $A \beta$ increases influx of $\mathrm{Ca}^{2+}$ into cells as well as release from the endoplasmic reticulum. ${ }^{27}$ Furthermore, $A \beta$ suppresses VDR expression, and increases expression of $\mathrm{Ca}_{\mathrm{v}} 1.2$ (a subunit of LVSCC). ${ }^{32}$ Conversely, vitamin $D$ decreases expression of $A \beta$ precursor protein (APP). ${ }^{33}$

\section{Oxidative stress}

Oxidative stress is known to induce apoptosis, which is a key component in neurodegenerative diseases. ${ }^{34}$ Therefore, antioxidants have an essential role in the brain where high oxygen consumption produces free radicals, e.g., reactive oxygen species (ROS). ${ }^{35}$ Oxidative stress markers have been found to be associated with $A D$ and mixed dementia. ${ }^{36}$ Many genes responsible for redox homeostasis are under the control of vitamin $D$ and VDR/RXR. Expression of redox-sensitive transcription factor, nuclear factor erythroid 2-related factor 2 (TNrf2) is under control of vitamin D. TNrf2 is activated in response to increased levels of ROS and acts to increase the expression of many antioxidants, as well as feeding back to increase VDR/RXR expression. ${ }^{27}$ Vitamin D regulation of klotho expression, an antiaging gene, also reduces oxidative stress. ${ }^{37}$ Furthermore, expression of $k$ lotho is inversely correlated with $A \beta$ accumulation, tau hyperphosphorylation, 
Figure 1: Vitamin D acquisition, metabolism and modes of action

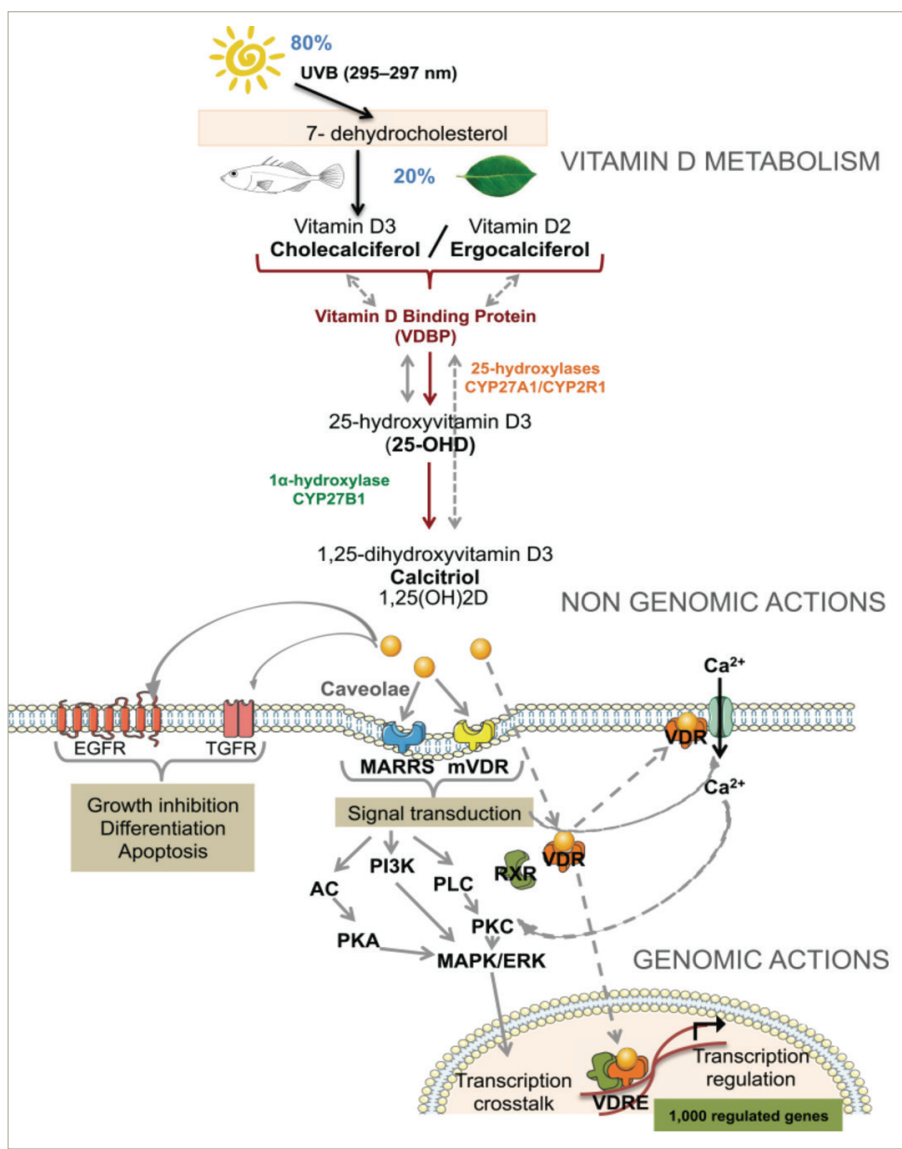

Cholecalciferol or vitamin D3 and ergocalciferol or vitamin D2 follow the same metabolic pathway. Blood metabolites include 25(OH)D produced by the liver, which is bound to vitamin D binding protein. Renal and extra-renal CYP27B1 (1 $\alpha$-hydroxylase) produces the active metabolite 1,25(OH)2D. 25(OH)D or 1,25(OH)2D enters the cell through passive diffusion or megalin-dependent transport. Once inside the cell, 1,25(OH)2D binds to its nuclear receptor VDR and after dimerization with $R X R$, forms a regulatory complex which can bind target genes that contain a vitamin D responsive element (VDRE). 1,25(OH)2D can also induce rapid non-genomic responses by binding to its membrane receptor (MARRS) or a membrane VDR and regulate the activity of adenylate cyclase, PLC, and PKC proteins. 1,25(OH)2D also induces modulation of calcium release from intracellular stores and can interact with TGF and EGF receptors to modulate cell cycle processes. These different modes of action and the crosstalks operated by vitamin D signaling ultimately lead to transcription modulation of hundreds of genes, depending on the celltype considered. Reproduced with permission from Landel et al., 2016. ${ }^{77}$

$A C=$ adenylate cyclase; $E G F R=$ epidermal growth factor receptor; $M A R R S=$ membraneassociated rapid response steroid-binding receptor; $m V D R=$ membrane vitamin $D$ receptor; $P I 3 K=$ phosphoinositide 3 -kinase; $P L C=$ phospholipase $C ; P K A=$ protein kinase $A ; P K C=$ protein kinase $C ; R X R=$ retinoid $\times$ receptor; TGFR $=$ transforming growth factor receptor; $U V B=$ ultraviolet $B ; V D B P=$ vitamin $D$ binding protein; $V D R=$ vitamin $D$ receptor; $V D R E=$ vitamin $D$ responsive element.

and the AD phenotype in mice. ${ }^{38}$ The receptor for advanced glycation endproduct (RAGE) is an enzyme whose expression is decreased by vitamin D. The RAGE is elevated in AD and contributes to oxidative stress by activating nicotinamide adenine dinucleotide phosphate (NADPH) oxidase (NOX), a mitochondrial enzyme involved in ROS production. ${ }^{27}$ Glutathione (GSH) is a major antioxidant molecule in the brain; depletion of GSH is implicated in both $A D$ and PD. ${ }^{35}$ Decreases in GSH are associated with enhanced transient receptor potential melastatin type 2 (TRPM2) function in hippocampal pyramidal neurons leading to further $\mathrm{Ca}^{2+}$ dysregulation. ${ }^{39}$ Increases in $\mathrm{Ca}^{2+}$ increase mitochondrial production of ROS. ${ }^{27}$ Increases in ROS sensitize RYRs, exacerbating the increases in $\mathrm{Ca}^{2+}$, and therefore contributing to impairment of memory formation and retention through decreases in the slow component of afterhyperpolarization and LTD, respectively. ${ }^{30}$ Vitamin D upregulates expression of both glutamate cysteine ligase (GCL), which is responsible for GSH production, and glutathione reductase (GR), which reduces glutathione disulfide (GSSG) back to GSH, thereby reducing oxidative stress. ${ }^{40,41}$

The repetitive surges in $\mathrm{Ca}^{2+}$ in the dopaminergic neuronal pacemaker mechanism in the substantia nigra pars compacta (SNC) predispose it to $\mathrm{Ca}^{2+}$ dysregulation leading to cell death via mitochondrial stress and production of ROS. The $\mathrm{Ca}^{2+}$ surges are mediated by LVSCCS, the expression of which is regulated by vitamin $\mathrm{D}$. Therefore, the observed reduction of oxidative stress in the SNC from vitamin D supplementation can be explained by reduction in $\mathrm{Ca}^{2+}$ levels from decreased LVSCC expression. This preventative role of vitamin $D$ in $P D$ is further supported by the finding that a particular single-nucleotide polymorphism (SNP) in the CACNA1C gene (codes for a subunit of LVSCC) is associated with PD, but only when vitamin D deficiency is also present. ${ }^{27,31,42}$ In addition to $\mathrm{Ca}^{2+}$ and redox homeostasis, vitamin $\mathrm{D}$ has been shown to regulate cellular homeostasis by increasing markers of autophagy, thereby inhibiting the PD process in both 1-methyl-4-phenyl1,2,3,6-tetrahydropyridine (MPTP) and rotenone models of induced PD. ${ }^{43,44}$

\section{Immune modulatory and neurotrophic role}

Pro-inflammatory state increases with age, contributing to cognitive decline and neurodegenerative disorders of the brain. Vitamin D is integral to regulating the central nervous system (CNS) immune system and mitigating these effects. ${ }^{45}$ Microglia, which are an essential part of the immune system in the brain, express CYP27B1 to synthesize calcitriol $(1,25[\mathrm{OH}] 2 \mathrm{D})$ from calcifediol $(25[\mathrm{OH}] 2)$. Vitamin D promotes the expression of interleukin (IL)-10 by microglia, which acts by an intracrine mechanism to increase expression of suppressor of cytokine signaling-3 (SOCS3). SOSCS3 inhibits expression of pro-inflammatory cytokines (IL-6, IL-12 and tumor necrosis factor alpha [TNF- $\alpha]) .{ }^{46} 1,25(\mathrm{OH}) 2$ vitamin D supplementation also inhibits the secretion of pro-inflammatory cytokines monocyte chemotactic protein-1 (MCP-1) and IL-8 by monocytes..$^{40}$ Recent transcriptomic analysis and a genome-wide analysis of VDR binding sites suggest that IL-8 expression is under the primary control of vitamin D. ${ }^{47,48}$ Dendritic cells with higher available calcidiol (25[OH] D3) are able to influence T cell response to favor regulatory $\mathrm{T}$ cell differentiation and reduced inflammatory response from $\mathrm{T}$ cells. ${ }^{49} \mathrm{~A}$ key component of PD pathology is neuroinflammation, specifically the release of ROS by activated microglia causing both neurotoxicity and oligomerization of $\alpha$-synuclein (Lewy body). ${ }^{50}$ Vitamin $D$ supplementation has been shown to significantly reduce ROS production by monocytes. ${ }^{40}$ Vitamin $\mathrm{D}$ also inhibits the production of nitric oxide by activated microglial cells, further preventing neuronal cell damage..$^{51}$ Brain pericytes respond to vitamin $D$ by increasing expression of genes that inhibit neuroinflammation, mitigating the age-related inflammatory changes in the hippocampus that are pathological in AD. Additionally, pericytes respond to TNF- $\alpha$ and interferon- $\gamma$ by inducing transcription of CYP27B1. ${ }^{52}$ Microglial overproduction of IL-1 $\beta$ leads to cognitive decline by inhibition of brainderived neurotrophic factor (BDNF), thereby interfering with hippocampal synaptic plasticity and LTP. ${ }^{53}$ Vitamin D has been shown to mitigate the proinflammatory state by increasing IL-10 and decreasing IL-1 $\beta$ expression, which is correlated with decreasing amyloid burden. ${ }^{45}$ This relationship with pro-inflammatory cytokines has been shown to be significant only in non-carriers of apolipoprotein E (APOE)- $\varepsilon 4$, while carriers of APOE- $\varepsilon 4$ who have $A D$ have a positive relationship between vitamin $D$ levels and 
pro-inflammatory cytokines. ${ }^{54}$ Vitamin D also induces nerve growth factor release in cortical neurons, which confers protective effects and has been shown to decrease expression of APP. ${ }^{31,55}$

Vitamin $\mathrm{D}$ also appears to have an important impact on VaD by reducing the likelihood of secondary immune-mediated injury to the CNS caused by stroke or small vessel disease. Indeed, early work demonstrated that ischemic injury caused by infarction is reduced with vitamin D supplementation in rats, which is attributed to increased expression of glial cell line-derived neurotrophic factor (GDNF)..$^{56}$ When compared with patients with higher vitamin D levels, patients with lower vitamin D levels at the time of ischemic strokes had larger ischemic infarct volumes, increased levels of cognitive impairment, and decreased improvement in function during rehabilitation. ${ }^{57,58}$

\section{Synaptic role}

Vitamin D3 supplementation has been shown to increase neuronal excitability in the hippocampus of rats - loss of neuronal excitability occurs with aging and is a proposed cause of cognitive decline. Vitamin D also upregulated multiple genes that were essential for synaptic plasticity (synaptojanin 1 and synaptotagmin 2, calcium/calmodulin-dependent protein kinase $\| \delta[$ [CaMKII $\delta]$ ), as well as those for normal synaptic functioning (receptors for several major neurotransmitters including dopamine, glutamate, and serotonin receptors). ${ }^{59}$

Dysfunction of the substantia nigra seen in PD has been attributed to a decrease in tyrosine hydroxylase $(\mathrm{TH})$ and dopamine synthesis. ${ }^{60}$ It has been observed that vitamin D supplementation increases TH expression and dopamine production in dopaminergic neurons. The study also shows that vitamin D upregulates $\mathrm{N}$-cadherin which plays a role in neurogenesis of dopamine neurons as well as in synaptic plasticity and memory. ${ }^{61,62}$

$1,25(\mathrm{OH}) \mathrm{D}$ treatment has been shown to increase the activity of choline acetyltransferase activity and increase acetylcholine levels in brain areas relevant to $A D$. Type 2 diabetes, a major risk factor for cognitive decline and $A D$, is thought to induce reduction in acetylcholine levels seen in $A D$. An improvement in cognitive decline related to type 2 diabetes with vitamin D supplementation has been observed in animal (rat) models mediated by increased choline acetyltransferase activity and a decrease in acetylcholinesterase activity.

Glutathione can act as a neurotransmitter and neuromodulator. It is thought to confer protection against glutamate excitotoxicity by acting on glutamate receptors. Glutathione levels are increased by vitamin D supplementation, and thus it has been demonstrated that vitamin $\mathrm{D}$ supplementation can prevent the glutamate toxicity implicated in cognitive decline. ${ }^{65}$

Along with the previously mentioned regulation of dopamine, vitamin D responsive elements (VDREs) are found in tryptophan hydroxylase (TPH) promoter regions, and 1,25(OH)D increased expression of TPH2 mRNA (messenger RNA) in the brain, implying vitamin D control over serotonin levels in the brain. ${ }^{66}$ Indeed, 1,25(OH)D treatment prevented both dopamine and serotonin depletion in certain areas of the brain. ${ }^{67}$

\section{Genetic connection}

Allelic differences in genes related to vitamin $D$ status and normal functions of vitamin $\mathrm{D}$ have been implicated in cognitive decline, $\mathrm{AD}$, and $\mathrm{PD} .{ }^{10}$ Certain
SNPS in the gene for vitamin D binding protein (VDBP) have been shown to correlate with both lower serum vitamin D levels and cognitive decline with age. ${ }^{10}$

Incidence of PD has been shown to be increased in individuals with certain polymorphisms in the VDR gene. However, the findings that support this are variable in respect to the particular sites that are related to the risk of PD. Polymorphisms at the Fokl site on VDR was associated with PD across multiple studies. ${ }^{18-20,68,69} \mathrm{Bsml}$ polymorphisms were also associated with PD susceptibility in two meta-analyses, one of which specified that this correlation was only found in the Asian population studied..$^{19,20}$ Polymorphisms in Taql and Apal were generally shown to not be associated with PD with two exceptions: one study of 382 patients with PD and 242 healthy controls found a correlation between Apal genotype and PD; another study on individuals with high lifetime ultraviolet exposure found that homozygotes of particular Taql and Apal alleles had a substantial and significant decreased risk of PD. ${ }^{18,21}$ The former of the previous two studies also found that genotype of VDBP confers a risk for PD. ${ }^{18}$

Vitamin $D$ receptor polymorphisms are also associated with the risk of AD. Apal genotype was found to be a risk factor in two original studies and one meta-analysis. ${ }^{17,19,70}$ The aforementioned meta-analysis also found a relationship between Taql genotype and $A D .^{19}$ In other studies, a direct relationship with Taql was not found; however one study did observe that the haplotype of Apal and Taql did demonstrate a correlation with AD susceptibility. Similarly, other SNP sites studied did not individually demonstrate a link to $A D$, but the haplotype of five sites including Apal, Taql, Bsml, Tru9l, and Fokl (which the authors titled TaubF) did demonstrate a significant relationship in one study despite the latter three sites having no individual significance in this regard. ${ }^{16}$ An allele of the binding site of the transcription factor CDX-2 on the VDR gene is associated with lower promoter activity and correlated with late-onset AD. ${ }^{33}$

A known risk factor for AD is genotype of APOE. Specifically, the APOE- 44 allele is known to confer a risk for $A D$. One study demonstrated that noncarriers of APOE- $\varepsilon 4$ had a negative correlation between pro-inflammatory cytokines and vitamin D versus a positive correlation in carriers of APOE- $\varepsilon 4$. This implies that hypovitaminosis D confers a greater risk for $A D$ in those without this genetic predisposition. ${ }^{54}$

\section{Other mechanisms}

In addition to the mechanisms discussed previously, vitamin D is involved in several other processes related to AD. Hypovitaminosis D has even been associated with subjective cognitive complaint, an early predictor of MNDS and $A D .{ }^{71} 1 \alpha, 25(\mathrm{OH}) 2$-vitamin $\mathrm{D} 3$ has been shown to induce clearance of $A \beta$ and reduction of amyloid burden through phagocytosis by macrophages and efflux across the blood-brain barrier (BBB). ${ }^{22,73}$ Efflux is thought to be mediated through induction of P-glycoprotein expression by vitamin D. ${ }^{74}$ Two recent studies have implicated vitamin $D$ in the regulation of $A \beta$ production based on mRNA transcriptional analysis demonstrating vitamin D transcription regulation of genes involved in APP processing, as well as co-localization of VDR on neuronal cell membranes with APP and enzymes involved in APP processing. ${ }^{75,76}$ Recent transcriptomic analysis also found vitamin D/VDR regulation of estrogen receptor 1 (ESR1) and insulin growth factor 1 (IGF1), and suggests a role for these two signaling pathways in preventing brain aging and neurodegenerative disease through neuronal survival, synaptic plasticity, and against $A \beta$ toxicity. ${ }^{47}$ This mechanism 
involving estrogen could also explain why there appears to be a much stronger connection between vitamin $D$ and $A D$ in females. ${ }^{77}$ It is proposed that klotho is responsible for inhibiting the insulin/IGF1 signaling pathway, thereby preventing aging. This idea is supported by the fact that vitamin $D$ reverses insulin signaling disruption in a model of $A D .^{78}$

In addition to genetic impacts of vitamin D/VDR, vitamin D also acts via the membrane-associated rapid response steroid-binding receptor (MARRS). In mice, activation of MARRS was shown to induce cognitive improvements and increased axon density in the medial prefrontal and perirhinal cortices, inducing cognitive improvements. ${ }^{79}$

\section{Treatment implications}

\section{Alzheimer's disease}

Recent consensus on the role of vitamin $D$ in $A D$ determined that because hypovitaminosis $D$ is considered to be etiological for $A D$, it should be screened for and corrected in patients with cognitive impairment. ${ }^{80}$ An early prospective study showing lower $A D$ among women with higher dietary vitamin D intake at 7-year follow-up, showed consuming $\sim 800 \mathrm{IU} /$ day divided $\mathrm{AD}$ risk by five. ${ }^{81}$ In patients with $A D$, vitamin $\mathrm{D}$ may be a useful standalone treatment, as it was shown to increase $A \beta$ clearance in a recent randomized controlled trial (RCT)..$^{82}$ Another $\mathrm{RCT}$ using adults with dementia found significant improvement over a 3- and 6-month period for patients being supplemented with vitamin D versus those patients who were not supplemented. ${ }^{83}$ One RCT showed significant benefit to using vitamin D in tandem with a standard therapy - memantine - demonstrating greater benefit and potentiating effects of using the two agents together. ${ }^{84}$ Another study using animal models (mice) of AD demonstrated that combined vitamin $\mathrm{D}$ and resveratrol (an antioxidant) treatment was more effective than vitamin $\mathrm{D}$ alone in preventing neuronal degeneration. ${ }^{78}$ Vitamin D was also shown to improve cognition, cholinergic transmission, and episodic memory in diabetic rats. ${ }^{64}$ An important study recently showed the first therapeutic program to reverse cognitive decline in $A D$. The treatment was customized for each individual patient and considered both genetic and metabolic markers in the design of treatment programs. Vitamin D status was considered, and vitamin D supplementation was included in multiple cases. ${ }^{85}$ Further studies are needed to evaluate the efficacy of vitamin D, and what concurrent treatments work best with it.

\section{Parkinson's disease}

Despite mounting evidence of a causal role of hypovitaminosis D in PD, there has only been one randomized placebo-controlled trial using vitamin D supplementation in PD. The 12-month study measured the disease with the modified HY scale (Hoehn and Yahr scale: a measure of disease progression which outlines the stages of PD in a one-to-five scale, with one being "Unilateral involvement only" and five being "Wheelchair bound or bedridden unless aided"), the UPDRS (Unified Parkinson's Disease Rating Scale: a six-part evaluation of the progression of PD, which includes the HY scale), the PDQ39 (Parkinson's Disease Questionnaire-39: an assessment of quality of life in PD), and the MMSE (Mini-Mental State Examination: a 30-point questionnaire commonly used to evaluate cognitive impairment). All scores were assessed while patients were on concurrent standard therapy, namely levodopa. The results showed significantly more increase on the HY scale in the control group (0.33) versus the vitamin D group (0.02). Part II scores of the UPDRS, which is a self-evaluation of the activities of daily life, were found to remain unchanged in the vitamin D group, but became significantly worse in the placebo group. On the other hand, the rest of the parts of the UPDRS, as well as the MMSE were not different within groups or between groups. Importantly, the study also found that when comparing the results with specific genotypes for the VDR gene, these differences were only significant in patients with the FokI TT and CT genotype, but not the CC genotype. ${ }^{86}$ There is a clear need for further study in this area.

\section{Vascular dementia}

The effects of vitamin D in VaD are still controversial. However, multiple studies have found an association between hypovitaminosis $D$ and $\operatorname{VaD}$ as outlined below. Vitamin D insufficiency has been shown to increase the risk of VaD; furthermore, the risk for those with hypertension and hypovitaminosis $D$ was much greater than for the risk increase from hypertension alone in the older adult Asian-Indian population. ${ }^{9}$ An association has also been found between serum 25(OH)D level and cerebral small vessel disease - a common cause of cognitive dysfunction in older adults. ${ }^{24}$ Additionally, there is an association between serum $25(\mathrm{OH})$ D level and micro- and macrovascular events in patients with type 2 diabetes. ${ }^{25}$ Recent transcriptomic analysis has shown some regulation of expression of genes involved in both vascular and endothelial processes. ${ }^{47}$ Regarding vitamin D as a treatment, one study has shown vitamin D given in combination with memantine prevents axonal degeneration caused by exposure to lysed blood as is the case with intracranial hemorrhage. ${ }^{87}$

\section{General considerations}

With new emphasis on the importance of maintaining appropriate vitamin D status, there has been an increase in the use of vitamin D supplements. Unfortunately, this has also led to an increase in cases of vitamin D intoxication, with one review finding $75 \%$ of all reports being published since $2010 .{ }^{8}$ Vitamin D intoxication is uncommon overall, but it can lead to hypercalcemia and hypercalciuria with fatal outcomes possible in extreme cases; therefore special consideration should be taken when correcting vitamin D deficiency. However, vitamin D intoxication is rare, and typically only occurs when very large doses are taken for an extended period of time. One review noted that there were no recorded cases of vitamin $D$ intoxication at oral intake less than 30,000 IU/day. ${ }^{89}$ Therefore, based on recommendations on vitamin D supplementation from the American Geriatrics Society, we recommend a starting dose of 1,000 IU/day to treat vitamin D deficiency in older adults which should be titrated up based on serum measurements. ${ }^{90}$

\section{Conclusion}

Vitamin D deficiency is very common in older adults for a variety of reasons, as discussed above. Accumulating evidence shows that vitamin D plays a central role in calcium homeostasis, neurotransmission, $A \beta$ and tau accumulation, oxidative stress, and immune regulation. Hypovitaminosis D may cause dysfunction of the physiological mechanisms listed above, thereby contributing to cognitive decline with old age, $A D, V a D$, and PD. Many observational studies and preclinical studies reported direct associations between decreased $25(\mathrm{OH})$ vitamin $\mathrm{D}$ concentrations and MNDS. Although evidence from randomized placebo-controlled trials is scarce, several trials have reported cognitive improvements among older adults supplemented with vitamin D. Further evidence from well designed and adequately powered clinical trials is required to establish the role of vitamin D in preventing or treating MNDs in older adults. Based on the current evidence we recommend as a minimum a sensible increase in vitamin D intake in geriatric patients, or, more extensively, monitoring and correction of serum vitamin D levels, especially in those afflicted by, or at risk of, neurocognitive disorders. $\square$ 
1. Grønli $O$, Kvamme JM, Jorde $R$, Wynn R. Vitamin $D$ deficiency is common in psychogeriatric patients, independent of diagnosis. BMC Psychiatry. 2014;14:134.

2. Holick MF, Chen TC. Vitamin D deficiency: a worldwide problem with health consequences. Am J Clin Nutr. 2008;87:1080S-1086S

3. Forrest KYZ, Stuhldreher WL. Prevalence and correlates of vitamin D deficiency in US adults. Nutr Res. 2011:31:48-54.

4. Afzal S, Brøndum-Jacobsen P, Bojesen SE, Nordestgaard BG. Genetically low vitamin D concentrations and increased mortality: mendelian randomisation analysis in three large cohorts. BMJ. 2014;349:86330.

5. Littlejohns TJ, Henley WE, Lang IA, et al. Vitamin D and the risk of dementia and Alzheimer disease Neurology 2014:83:920-8.

6. Annweiler C, Fantino B, Schott AM, et al. Vitamin D insufficiency and mild cognitive impairment: cross-sectional association. Eur Neurol. 2012:19:1023-9.

7. Shen L, Ji HF. Associations between vitamin D status, supplementation, outdoor work and risk of Parkinson's disease: meta-analysis assessment. Nutrients. 2015;7:4817-27.

8. Sleeman I, Aspray T, Lawson R, et al. The role of vitamin D in disease progression in early Parkinson's disease. J Parkinsons Dis. 2017;7:669-75.

9. Prabhakar P, Chandra SR, Supriya M, et al. Vitamin D status and vascular dementia due to cerebral small vessel disease in the elderly Asian Indian population. J Neurol Sci. 2015;359:108-11.

10. Kueider AM, Tanaka T, An Y, et al. State- and trait-dependent associations of vitamin-D with brain function during aging. Neurobiol Aging. 2016;39:38-45

11. Kużma $\mathrm{E}$, Soni $\mathrm{M}$, Littlejohns $\mathrm{TJ}$, et al. Vitamin $\mathrm{D}$ and memory decline: two population-based prospective studies. J Alzheimers Dis. 2016;50:1099-108.

12. Littlejohns TJ, Kos K, Henley WE, et al. Vitamin D and risk of neuroimaging abnormalities. PloS One. 2016;11:e0154896.

13. Darwish $H$, Zeinoun $P$, Ghusn $H$, et al. Serum 25-hydroxyvitamin D predicts cognitive performance in adults. Neuropsychiatr Dis Treat. 2015;11:2217-23.

14. Shrestha S, Lutsey PL, Alonso A, et al. Serum 25-hydroxyvitamin D concentrations in mid-adulthood and Parkinson's disease risk. Mov Disord. 2016; 31:972-8.

15. Karakis I, Pase MP, Beiser A, et al. Association of serum vitamin $D$ with the risk of incident dementia and subclinical indices of brain aging: The Framingham Heart Study. J Alzheimers Dis. 2016;51:451-61.

16. Gezen-Ak D, Dursun E, Bilgiç B, et al. Vitamin D receptor gene haplotype is associated with late-onset Alzheimer's disease Tohoku J Exp Med. 2012;228:189-96.

17. Gezen-AK D, Dursun E, Ertan T, et al. Association between vitamin $D$ receptor gene polymorphism and Alzheimer's disease. Tohoku J Exp Med. 2007;212:275-82.

18. Gezen-Ak D, Alaylıoğlu M, Genç G, et al. GC and VDR SNPS and vitamin D levels in Parkinson's disease: the relevance to clinical features. Neuromolecular Med. 2017:19:24-40

19. Lee YH, Kim J-H, Song GG. Vitamin D receptor polymorphisms and susceptibility to Parkinson's disease and Alzheimer's disease: a meta-analysis. Neurol Sci. 2014;35:1947-53.

20. Li C, Qi H, Wei S, et al. Vitamin D receptor gene polymorphisms and the risk of Parkinson's disease Neurol Sci. 2015:36:247-55.

21. Gatto NM, Sinsheimer JS, Cockburn M, et al. Vitamin D receptor gene polymorphisms and Parkinson's disease in a population with high ultraviolet radiation exposure. I Neurol SCi. 2015;352:88-93.

22. Peterson AL, Murchison C, Zabetian C, et al. Memory, mood, and vitamin D in persons with Parkinson's disease. J Parkinsons Dis. 2013;3:547-55.

23. Gatto NM, Paul KC, Sinsheimer IS, et al. Vitamin D receptor gene polymorphisms and cognitive decline in Parkinson's disease. Neurol SCi. 2016;370:100-6.

24. Chung PW, Park KY, Kim JM, et al. 25-hydroxyvitamin D status is associated with chronic cerebral small vessel disease. Stroke. 2015;46:248-51.

25. Herrmann $M$, Sullivan $D R$, Veillard AS, et al. Serum 25-hydroxyvitamin D: a predictor of macrovascular and microvascular complications in patients with type 2 diabetes. Diabetes Care. 2015;38:521-8.

26. Afzal S, Nordestgaard BG. Vitamin D, hypertension, and ischemic stroke in 116655 individuals from the general population: a genetic study. Hypertension. 2017;70:499-507.

27. Berridge MJ. Vitamin D cell signalling in health and disease. Biochem Biophys Res Commun. 2015;460:53-71.

28. Foster TC. Calcium homeostasis and modulation of synaptic plasticity in the aged brain. Aging Cell. 2007;6:319-25

29. Foster TC, Sharrow KM, Masse JR, et al. Calcineurin links $\mathrm{Ca}^{2+}$ dysregulation with brain aging. J Neurosci. 2001;21:4066-73.

30. Bodhinathan $\mathrm{K}$, Kumar A, Foster TC. Redox sensitive calcium stores underlie enhanced after hyperpolarization of aged neurons: role for ryanodine receptor mediated calcium signaling. I Neurophysiol. 2010;104:2586-93.

31. Gezen-Ak D, Dursun E, Yilmazer S. The effects of vitamin D receptor silencing on the expression of LVSCC-A1C and LVSCCA1D and the release of NGF in cortical neurons. Plos One. A1D and the re11;6:e17553.

32. Berridge MJ. Calcium regulation of neural rhythms, memory and Alzheimer's disease. J Physiol. 2014;592:281-93.

33. Wang L, Hara K, Van Baaren JM, et al. Vitamin D receptor and Alzheimer's disease: a genetic and functional study. Neurobio
Aging. 2012;33:1844.e1-1844.e9.

34. Johnson WM, Wilson-Delfosse AL, Mieyal JJ. Dysregulation of glutathione homeostasis in neurodegenerative diseases Nutrients. 2012:4:1399-440.

35. Gu F, Chauhan V, Chauhan A. Glutathione redox imbalance in brain disorders. Curr Opin Clin Nutr Metab Care. 2015;18:89-95.

36. Hatanaka $\mathrm{H}$, Hanyu $\mathrm{H}$, Fukasawa R, et al. Differences in periphera oxidative stress markers in Alzheimer's disease, vascular dementia and mixed dementia patients. Geriatr Gerontol Int. 2015;15:53-8.

37. Wang Y, Sun Z. Current understanding of klotho. Ageing Res Rev. 2009;8:43-51.

38. Kuang $X$, Chen $Y-S$, Wang $L F$, et al. Klotho upregulation contributes to the neuroprotection of ligustilide in an Alzheimer's disease mouse model. Neurobiol Aging. 2014:35:169-78.

39. Belrose JC, Xie YF, Gierszewski LJ, et al. Loss of glutathione homeostasis associated with neuronal senescence facilitates TRPM2 channel activation in cultured hippocampal pyramidal neurons. Mol Brain. 2012;5:11

40. Jain SK, Micinski D. Vitamin D upregulates glutamate cysteine ligase and glutathione reductase, and GSH formation, and decreases ROS and MCP-1 and IL-8 secretion in high-glucose exposed U937 monocytes. Biochem Biophys Res Commun. 2013:437:7-11.

41. Alvarez JA, Chowdhury R, Jones DP, et al. Vitamin D status is independently associated with plasma glutathione and cystein thiol/disulphide redox status in adults. Clin Endocrinol (Oxf). 2014;81:458-66.

42. Wang $L$, Maldonado $L$, Beecham GW, et al. DNA variants in CACNA1C modify Parkinson disease risk only when vitamin D level is deficient. Neurol Genet. 2016;2:e72.

43. $\mathrm{L} \mathrm{H}$, Jang $\mathrm{W}$, Kim $\mathrm{HJ}$, et al. Biochemical protective effect of 1,25-dihydroxyvitamin D3 through autophagy induction in the MPTP mouse model of Parkinson's disease. Neuroreport. 2015;26:669-74.

44. Jang W, Kim HJ, Li H, et al. 1,25-Dyhydroxyvitamin $\mathrm{D}_{3}$ attenuates rotenone-induced neurotoxicity in SH-SY5Y cells through induction of autophagy. Biochem Biophys Res Commun. 2014;451:142-7.

45. Briones TL, Darwish H. Vitamin D mitigates age-related cognitive decline through the modulation of pro-inflammatory state and decrease in amyloid burden. J Neuroinflammation. 2012;9:244.

46. Boontanrart M, Hall SD, Spanier JA, et al. Vitamin D3 alters microglia immune activation by an IL-10 dependent SOCS3 mechanism. J Neuroimmunol. 2016;292:126-36.

47. Landel $\mathrm{V}$, Millet $\mathrm{P}$, Baranger $\mathrm{K}$, et al. Vitamin D interacts with Esr1 and Igf1 to regulate molecular pathways relevant to Alzheimer's disease. Mol Neurodegener. 2016;11:22.

48. Ryynänen J, Carlberg C. Primary 1,25-dihydroxyvitamin D3 response of the interleukin 8 gene cluster in human monocyteand macrophage-like cells. PloS One. 2013;8:e78170.

49. Jeffery LE, Wood AM, Qureshi OS, et al. Availability of 25-hydroxyvitamin D(3) to APCs controls the balance between regulatory and inflammatory $\mathrm{T}$ cell responses. I Immunol. 2012:189:5155-64.

50. Blesa J, Trigo-Damas I, Quiroga-Varela A, Jackson-Lewis VR. Oxidative stress and Parkinson's disease. Front Neuroanat. 2015;9:91.

51. Hur J, Lee P, Kim MJ, Cho YW. Regulatory effect of 25-hydroxyvitamin D3 on nitric oxide production in activated microglia. Korean J Physiol Pharmacol. 2014;18:397-402.

52. Nissou MF, Guttin A, Zenga C, et al. Additional clues for a protective role of vitamin $\mathrm{D}$ in neurodegenerative diseases: 1,25-dihydroxyvitamin D3 triggers an anti-inflammatory response in brain pericytes. J Alzheimers Dis. 2014;42:789-99.

53. Patterson SL. Immune dysregulation and cognitive vulnerability in the aging brain: Interactions of microglia, IL- $1 \beta$, BDNF and synaptic plasticity. Neuropharmacology. 2015;96:11-8.

54. Dursun E, Alaylıoglu M, Bilgiç B, et al. Vitamin D deficiency might pose a greater risk for $A p 0 E \varepsilon 4$ non-carrier Alzheimer's disease patients. Neurol Sci. 2016:37:1633-43.

55. Banerjee A, Khemka VK, Ganguly A, et al. Vitamin D and Alzheimer's disease: neurocognition to therapeutics. Int $\mathrm{J}$ Alzheimers Dis. 2015;2015:192747.

56. Wang Y, Chiang Y-H, Su TP, et al. Vitamin D3 attenuates cortical infarction induced by middle cerebral arterial ligation in rats. Neuropharmacology. 2000;39:873-80.

57. Turetsky A, Goddeau Jr. RP, Henninger N. Low serum vitamin D is independently associated with larger lesion volumes after ischemic stroke. J Stroke Cerebrovasc Dis. 2015:24:1555-63.

58. Yalbuzdag SA, Sarifakioglu B, Afsar SI, et al. Is $25(\mathrm{OH}) \mathrm{D}$ associated with cognitive impairment and functional improvement in stroke? A retrospective clinical study. I Stroke Cerebrovasc Dis. 2015;24:1479-86

59. Latimer CS, Brewer LD, Searcy JL, et al. Vitamin D prevents cognitive decline and enhances hippocampal synaptic function in aging rats. Proc Natl Acad Sci U S A. 2014;111:E4359-66.

60. Zhu Y, Zhang J, Zeng Y. Overview of tyrosine hydroxylase in Parkinson's disease. CNS Neurol Disord Drug Targets. 2012:11:350-8.

61. Schrick C, Fischer A, Srivastava DP, et al. N-cadherin regulates cytoskeletally associated IQGAP1/ERK signaling and memory formation. Neuron. 2007; 55:786-98

62. Cui X, Pertile R, Liu P, Eyles DW. Vitamin D regulates tyrosine hydroxylase expression: $\mathrm{N}$-cadherin a possible mediator. Neuroscience. 2015;304:90-100

63. Sonnenberg J, Luine VN, Krey LC, Christakos S. 1,25-Dihydr oxyvitamin D3 treatment results in increased choline acetyltransferase activity in specific brain nuclei. Endocrinology. 1986;118:1433-9.

64. Alrefaie $Z$, Alhayani $A$. Vitamin $D_{3}$ improves decline in cognitive function and cholinergic transmission in prefrontal cortex of streptozotocin-induced diabetic rats. Behav Brain Res. 2015;287:156-62.

65. Annweiler C. Vitamin D in dementia prevention. Ann N Y Acad SCi. 2016;1367:57-63

66. Kaneko I, Sabir MS, Dussik CM, et al. 1,25-Dihydroxyvitamin D regulates expression of the tryptophan hydroxylase 2 and leptin genes: implication for behavioral influences of vitamin D. FASEB J. 2015;29:4023-35

67. Cass WA, Smith MP, Peters LE. Calcitriol protects against the dopamine- and serotonin-depleting effects of neurotoxic doses of methamphetamine. Ann N Y Acad Sci. 2006;1074:261-71.

68. Niu MY, Wang L, Xie AM. Apal, Bsml, Fokl, and Taql polymorphisms in the vitamin D receptor gene and Parkinson's disease. Chin Med $J(E n g)$ ). 2015;128:1809-14.

69. Tanaka K, Miyake Y, Fukushima W, et al. Vitamin D receptor gene polymorphisms, smoking, and risk of sporadic Parkinson's disease in Japan. Neurosci Lett. 2017:643:97-102.

70. Łaczmański Ł, Jakubik M, Bednarek-Tupikowska G, et al. Vitamin $D$ receptor gene polymorphisms in Alzheimer's disease patients. Exp Gerontol. 2015;69:142-7.

71. Tot Babberich EDN, Gourdeau C, Pointel S, et al. Biology of subjective cognitive complaint amongst geriatric patients: vitamin D involvement. Curr Alzheimer Res. 2015:12:173-8.

72. Hooshmand B, LökK J, Solomon A, et al. Vitamin D in relation to cognitive impairment, cerebrospinal fluid biomarkers, and brain volumes. J Gerontol A Biol Sci Med Sci. 2014;69:1132-8.

73. Mizwicki MT, Menegaz D, Zhang J, et al. Genomic and nongenomic signaling induced by $1 \alpha, 25(\mathrm{OH}) 2$-vitamin D3 promotes the recovery of amyloid- $\beta$ phagocytosis by Alzheimer's disease macrophages. J Alzheimers Dis. 2012;29:51-62.

74. Durk MR, Han K, Chow EC, et al. 1 $\alpha, 25$-Dihydroxyvitamin D3 reduces cerebral amyloid- $\beta$ accumulation and improves cognition in mouse models of Alzheimer's disease. I Neurosci. 2014:34:7091-101

75. Dursun $E$, Gezen-Ak D. Vitamin D receptor is present on the neuronal plasma membrane and is co-localized with amyloid precursor protein, ADAM10 or Nicastrin. PloS One. 2017; 12:e0188605

76. Gezen-Ak D, Atasoy IL, Candas E, et al. Vitamin D receptor regulates amyloid beta 1-42 production with protein disulfide isomerase A3. ACS Chem Neurosci. 2017:8:2335-46.

77. Landel V, Annweiler C, Millet P, et al. Vitamin D, cognition, and Alzheimer's disease: the therapeutic benefit is in the D-tails. J Alzheimers Dis. 2016;53:419-44.

78. Cheng J, Xia X, Rui Y, et al. The combination of $1 \alpha, 25$ dihydroxyvitaminD3 with resveratrol improves neuronal degeneration by regulating endoplasmic reticulum stress, insulin signaling and inhibiting tau hyperphosphorylation in SH-SY5Y cells. Food Chem Toxicol. 2016;93:32-40.

79. Tohda C, Lee YA, Goto Y, Nemere I. Diosgenin-induced cognitive enhancement in normal mice is mediated by $1,25 D_{3}$-MARRS. SC Rep. 2013;3:3395

80. Annweiler C, Dursun E, Féron F, et al. 'Vitamin D and cognition in older adults': updated international recommendations. J Intern Med. 2015;277:45-57.

81. Annweiler $C$, Rolland $Y$, Schott AM, et al. Higher vitamin D dietary intake is associated with lower risk of Alzheimer's disease: a 7-year follow-up. J Gerontol A Biol Sci Med Sci. 2012;67:1205-11.

82. Miller BJ, Whisner CM, Johnston CS. Vitamin D supplementation appears to increase plasma a 40 in vitamin D insufficient olde adults: a pilot randomized controlled trial. J Alzheimers Dis. 2016;52:843-7

83. Gangwar AK, Rawat A, Tiwari S, et al. Role of vitamin-D in the prevention and treatment of Alzheimer's disease. Indian J Physiol Pharmacol. 2015:59:94-9.

84. Annweiler C, Herrmann FR, Fantino B, et al. Effectiveness of the combination of memantine plus vitamin $D$ on cognition in patients with Alzheimer disease: a pre-post pilot study. $\operatorname{cog} n$ Behav Neurol. 2012;25:121-7.

85. Bredesen DE. Reversal of cognitive decline: a novel therapeutic program. Aging (Albany NY). 2014;6:707-17.

86. Suzuki M, Yoshioka M, Hashimoto M, et al. Randomized, doubleblind, placebo-controlled trial of vitamin D supplementation in Parkinson disease. Am J Clin Nutr. 2013;97:1004-13.

87. Charier D, Beauchet $\mathrm{O}$, Bell M, et al. Memantine plus vitamin D prevents axonal degeneration caused by lysed blood. ACS Chem Neurosci. 2015;6:393-7.

88. Taylor PN, Davies JS. A review of the growing risk of vitamin D toxicity from inappropriate practice. Br J Clin Pharmacol. 2018; DOl: 10.1111/bcp.13573.

89. Hathcock JN, Shao A, Vieth R, Heaney R. Risk assessment for vitamin D. Am J Clin Nutr. 2007;85:6-18

90. American Geriatrics Society Workgroup on Vitamin D Supplementation for Older Adults. Recommendations abstracted from the American Geriatrics Society consensus statement on vitamin $\mathrm{D}$ for prevention of falls and their consequences. J Am Geriatr SOC. 2014;62:147-52. 